\title{
Comparison of whole genome expression profile between preterm and full-term newborns
}

\author{
Przemko Kwinta ${ }^{1}$, Renata Bokiniec ${ }^{2}$, Mirosław Bik-Multanowski ${ }^{3}$, Clara-Cecilie Gunther ${ }^{4}$, \\ Agnieszka Grabowska ${ }^{3}$, Teofila Książek ${ }^{3}$, Anna Madetko-Talowska ${ }^{3}$, Katarzyna Szewczyk ${ }^{3}$, \\ Monika Szwarc-Duma2 ${ }^{2}$, Maria K. Borszewska-Kornacka², Lars O. Baumbusch ${ }^{5}$, \\ Cecilie Revhaug ${ }^{5}$, Ola D. Saugstad ${ }^{5}$, Jacek J. Pietrzyk ${ }^{1}$
}

${ }^{1}$ Department of Pediatrics Jagiellonian University Medical College, Cracow, Poland

${ }^{2}$ Neonatal and Intensive Care Department, Medical University of Warsaw, Poland

${ }^{3}$ Department of Medical Genetics, Jagiellonian University Medical College, Cracow, Poland

${ }^{5}$ Department of Pediatric Research, Oslo University Hospital and University of Oslo, Norway

\begin{abstract}
Objectives: Evaluate the time dependent expression of genes in preterm neonates and verify the influence of ontogenic maturation and the environmental factors on the gene expression after birth.

Material and methods: The study was carried out on 20 full-term newborns and 62 preterm newborns (mean birth weight $=1002$ [g] (SD: 247), mean gestational age $=27.2$ weeks (SD: 1.9)). Blood samples were drawn from all the study participants at birth and at the 36th week postmenstrual age from the preterm group to assess whole genome expression in umbilical cord blood and in peripheral blood leukocytes, respectively. (SurePrint G3 Human Gene Expression v3, 8x60K Microarrays (Agilent)).

Results: A substantial number of genes was found to be expressed differentially at the time of birth and at 36 PMA in comparison to the term babies with more genes being down-regulated than up-regulated. However, the fold change in the majority of cases was $<2.0$. Extremely preterm and very preterm infants were characterized by significantly down-regulated cytokine and chemokine related pathways. The number of down-regulated genes decreased and number of up-regulated genes increased at 36 PMA vs. cord blood. There were no specific gene expression pathway profiles found within the groups of different gestational ages.

Conclusions: Preterm delivery is associated with a different gene expression profile in comparison to term delivery. The gene expression profile changes with the maturity of a newborn measured by the gestational age.
\end{abstract}

Key words: transcriptome, very low birth weight, cord blood

Ginekologia Polska 2017; 88, 8: 434-441

\section{INTRODUCTION}

Progress in the development of intensive care achieved in the past few decades has resulted in an increased survival of the smallest infants. However, the improved survival of very low birth weight (VLBW) infants has led to an increased rate of complications, which is the underlying reason for a higher rate of infant mortality and morbidity [1-4].

Complications of prematurity such as bronchopulmonary dysplasia (BPD) or retinopathy of prematurity (ROP) are considered to be complex diseases and their pathogenesis depends on the interaction of a susceptible host with a multitude of environmental risk factors $[5,6]$. The most important risk factor for BPD or ROP is the degree of maturity of the newborn. However, the environmental factors like prenatal and postnatal infection, oxygen exposure, postnatal nutrition and weight gain, drugs and many others can modify the course of the disease $[7,8]$. Due to the complexity of these interactions studying the complications of 
prematurity is very difficult. The development of the tools that can evaluate many different genetic and environmental factors simultaneously is of crucial importance for future progress in our understanding of the pathogenesis and the pathomechanism of complications of prematurity.

Introduction of the microarray techniques to clinical studies was one of the most important turning points resulting in a dramatic progress in the field of human genetics during the last decade [9]. RNA-microarray allows analyzing the expression of all human genes. Computational methods of analyzing vast amounts of data are continuously being developed and quantitative tools for analyzing networks are now also more accessible. Microarrays can be used to study associations between genetic variations and occurrences of diseases, searching for cancer susceptibility markers, molecular karyotyping and pharmacogenomics [10]. The greatest advantage of this method is its ability to assess a great number of genetic factors and requires only a small amount of blood for testing, which is very important aspect when handling preterm neonates.

In this study the gene expression profiles in preterm infants born before 30 weeks of gestation were explored in order to evaluate the time dependent expressions of genes in preterm neonates and to verify whether the ontogenic maturation and environment factors can influence the genes expression after birth.

\section{MATERIAL AND METHODS}

A prospective study was conducted between September 1st, 2013, and November 30 $\mathrm{th}, 2015$. Two cohorts of children were collected: a preterm group and a control group that comprised term newborns. The enrollment of preterm children was divided into three subgroups: subgroup $1-$ newborns born $\leq 26$ weeks of gestation, subgroup 2 - newborns born between 27 and 28 weeks of gestation and subgroup 3 - newborns born between 29 and 30 weeks of gestation. The control group included healthy, full term newborns. All preterm children were born in the level III perinatal center and all control children were born in the level I city hospital.

Detailed perinatal history (birth weight, gestational age) and maternal history were recorded after birth. Appropriate Ethics Committee approved the study. An informed written consent was obtained from every parent before the birth of participant involved in the study.

\section{Microarray analysis}

Blood samples were drawn from all the study participants at birth and at the $36^{\text {th }}$ week postmenstrual age in the preterm group to assess the whole genome expression in umbilical cord blood and in peripheral blood leukocytes, respectively.
The density gradient centrifugation with Histopaque-1077 (Sigma-Aldrich) was performed for $30 \mathrm{~min}$, $2100 \mathrm{rpm}$ and at a room temperature. The interphase containing the mononuclear cells was collected to a new tube, washed two times in 1x PBS (Phosphate-Buffered Saline, 1x wo calcium and magnesium; Corning) followed by centrifugation $10 \mathrm{~min}, 1600 \mathrm{rpm}$, room temperature. Finally, pelleted MNCs (Mononuclear Cells) were suspended in 1x PBS solution. The final volume of the PBS suspension matched the starting volume of the processed blood $(0.25-0.95 \mathrm{~mL})$.

Subsequently, total RNA was extracted by RiboPure Blood Kit (Ambion, Thermo Fisher Scientific, Carlsbad, California, USA) and quantified by spectrophotometry (NanoDrop ND-1000; Thermoscientific, Waltham, USA). RNA quality and integrity was determined by 2100 Bioanalyzer (Agilent, Santa Clara, USA). For the single microarray experiment $50 \mathrm{ng}$ of total RNA was used.

To study the whole genome expression with a comprehensive coverage of genes and transcripts SurePrint G3 Human Gene Expression v3, 8x60K Microarrays (Agilent, Santa Clara, USA) were used. Microarray gene expression experiment was performed according to the manufacturer's protocol (Two-Color Microarray-Based Gene Expression Analysis — Low Input Quick Amp Labeling ver.6.9.1). After experiment, the arrays were scanned by SureScan Microarray Scanner (Agilent, Santa Clara, USA) and data was extracted using Feature Extraction Software (Agilent, Santa Clara, USA).

\section{Predefined comparisons}

The following gene expression comparisons were planned:

- newborns born $\leq 26$ weeks of gestation (extremely preterm) vs. full term,

- newborns born between 27-28 weeks of gestation (very preterm) vs. full term,

- newborns born between 29-30 weeks of gestation (moderately preterm) vs. full term,

- newborns born $\leq 26$ weeks of gestation (extremely preterm) vs. newborns born between 27-28 weeks of gestation (very preterm),

- newborns born $\leq 26$ weeks of gestation (extremely preterm) vs. newborns born between 29-30 weeks of gestation (moderately preterm),

- newborns born between 27-28 weeks of gestation (very preterm) vs. newborns born between 29-30 weeks of gestation (moderately preterm).

These comparisons were performed at two time points (at birth and at $36^{\text {th }}$ week postmenstrual age - PMA). Moreover, the gene expression profile at birth was compared to the gene expression profile assessed at the age of 36 weeks PMA for each preterm newborn. 


\section{Statistical analysis}

Quality control was performed by investigating Principal Component Analysis (PCA). None of the arrays were discarded due to poor quality. A moderated t-test was used to compare the differences between the groups [11]. Multiple testing correction was applied using the Benjamini-Hochberg procedure to control the false discovery rate (FDR). A probe set with a corrected for multiple comparisons $p$-value $<0.05$ was considered as significantly differentially expressed.

DAVID annotation tools were used to explore which predefined gene sets were significantly enriched between the study groups $[12,13]$. The KEGG (Kyoto Encyclopedia of Genes and Genomes; www.genome.jp/kegg) and Biocarta pathways (www.biocarta.com) were selected for analysis. Genes differentially expressed between the groups with $p$-values adjusted for multiple testing $<0.05$ and with fold change $(F C)>2.0$ were used as input for pathway enrichment analysis in DAVID.

\section{RESULTS}

Sixty-two preterm and twenty full-term newborns were included in the study. The mean birth weight of newborns in the preterm cohort was $1002 \mathrm{~g}$ (SD: 247), and the mean gestational age was 27.2 weeks (SD: 1.9 ). The clinical characteristics of the preterm subgroups are presented in Table 1. The control group included 10 girls and 10 boys, all with birthweight between 2500 and 4000 grams.

\section{Comparison of the cord blood gene expression profiles between preterm subgroups vs. full term newborns}

The number of significantly over- and under-expressed probes was 8362 in the extremely preterm children, 11180 in the very preterm and 8152 in the moderately preterm group. These numbers represent 5752, 7640 and 5745 genes, respectively. However, the fold change in majority of the cases was $<2$.0. Only genes with FC $\geq 2$ were used for pathway analysis and the results of these evaluations were presented in Table 2. The pathway enrichment analysis recognized only two pathways (hsa04060: Cytokine-cytokine receptor interaction and hsa04062: Chemokine signaling pathway), which were down-regulated in the group of extremely preterm infants compared to the group of full-term children. No pathway was up-regulated among extremely preterm children. Similar results were found in the group of very preterm children. The same two pathways were significantly down-regulated (hsa04060: Cytokine-cytokine receptor interaction and hsa04062: Chemokine signaling pathway) and none was up-regulated. In the group of moderately preterm children one pathway was up-regulated (hsa04612: Antigen processing and presentation) and no pathway was down-regulated.

Comparison between the gene expression profiles from peripheral blood leukocytes sampled at the age of 36th week PMA in the preterm children and from the umbilical blood of full-term infants revealed 9960, 12178 and

Table 1. The clinical characteristics of the population of preterm newborns

\begin{tabular}{|c|c|c|c|}
\hline & Extremely preterm $(n=19)$ & Very preterm $(n=23)$ & Moderately preterm $(n=20)$ \\
\hline Male/female & $8 / 11$ & $6 / 17$ & $9 / 11$ \\
\hline Birth weight (g); mean (SD) & $786(125)$ & $969(155)$ & $1246(211)$ \\
\hline Gestational age (weeks); mean (SD) & $25(1.0)$ & $27.5(0.5)$ & $29(1)$ \\
\hline Prenatal steroids & $14(74 \%)$ & $20(87 \%)$ & $14(70 \%)$ \\
\hline Oxygen at 28 day of life & $19(100 \%)$ & $14(60 \%)$ & $6(30 \%)$ \\
\hline Oxygen at $36 \mathrm{PMA}$ & $3(16 \%)$ & 0 & $1(5 \%)$ \\
\hline $\begin{array}{l}\text { Laser coagulation treatment due to } \\
\text { retinopathy of prematurity }\end{array}$ & $4(21 \%)$ & $1(4 \%)$ & 0 \\
\hline
\end{tabular}

PMA - postmenstrual age

Table 2. Comparison between gene expression profile from umbilical blood leukocytes sampled at birth in preterm children and in full-term infants

\begin{tabular}{|l|c|c|c|c|c|c|c|c|}
\hline & & \multicolumn{2}{|c|}{ Extremely preterm $(\mathbf{n}=\mathbf{1 9})$} & \multicolumn{2}{c|}{ Very preterm $(\mathbf{n}=\mathbf{2 3})$} & \multicolumn{2}{|c|}{ Moderately preterm $(\mathbf{n}=\mathbf{2 0})$} \\
\cline { 3 - 9 } & & Probes & Genes & Probes & Genes & Probes & Genes \\
\hline FC $>2.0$ & Up-regulated & 269 & 188 & 379 & 274 & 285 & 233 \\
\hline & Down-regulated & 504 & 362 & 755 & 562 & 347 & 275 \\
\hline
\end{tabular}

FC - fold change 
Table 3. Comparison between gene expression profiles from peripheral blood leukocytes sampled at the age of $36^{\text {th }}$ week postmenstrual age (PMA) in preterm children and from umbilical blood of full-term infants

\begin{tabular}{|l|c|c|c|c|c|c|c|c|}
\hline & & \multicolumn{2}{|c|}{ Extremely preterm $(\mathbf{n}=\mathbf{1 9})$} & \multicolumn{2}{|c|}{ Very preterm $(\mathbf{n}=\mathbf{2 3})$} & \multicolumn{2}{c|}{ Moderately preterm $(\mathbf{n}=\mathbf{2 0})$} \\
\cline { 3 - 8 } & & Probes & Genes & Probes & Genes & Probes & Genes \\
\hline FC $>2.0$ & Up-regulated & 596 & 377 & 328 & 204 & 274 & 165 \\
\hline FC $>2.0$ & Down-regulated & 113 & 75 & 115 & 83 & 87 & 55 \\
\hline
\end{tabular}

FC - fold change

9229 differentially expressed probes and 6573, 7893 and 6275 differentially expressed genes. The numbers of the most significantly differentially expressed probes and genes are presented in Table 3.

The pathway enrichment analysis did not show any differentially down-regulated pathways in the group of extremely preterm infants in comparison to the group of full-term children. However, hsa04668: TNF signaling pathway was up-regulated among the extremely preterm children. There were no down-regulated or up-regulated pathways found in the groups of very preterm children. The hsa04668: TNF signaling pathway was found up-regulated and no pathway was down-regulated in the moderately preterm infants.

\section{Comparison of the gene expression profiles at birth and at the age of $36^{\text {th }}$ week PMA}

The summary of the differentially expressed probes and genes between the birth time and the age of $36^{\text {th }}$ week PMA is presented in Table 4. In extremely preterm children, among the genes with a fold change $>2.0$, the expression of 538 probes (384 genes) decreased from birth to 36 weeks PMA and the expression of 457 probes ( 285 genes) increased between the two time points. No pathway was up-regulated at 36 PMA in comparison to the cord blood and 4 pathways were found down-regulated: hsa04510: Focal adhesion; hsa04611: Platelet activation; hsa04726: Serotonergic synapse and the hsa00590: Arachidonic acid metabolism pathway.

Table 4. The summary of the number of differentially expressed probes and genes between samples collected at birth and at the age of $36^{\text {th }}$ week PMA

\begin{tabular}{|l|l|l|l|l|}
\hline \multirow{2}{*}{} & \multicolumn{2}{|c|}{ Probes } & \multicolumn{2}{c|}{ Genes } \\
\cline { 2 - 6 } & Any FC & FC > 2.0 & Any FC & FC > 2.0 \\
\hline $\begin{array}{l}\text { Extremely preterm } \\
(n=19)\end{array}$ & 16862 & 995 & 10589 & 669 \\
\hline $\begin{array}{l}\text { Very preterm } \\
(n=23)\end{array}$ & 16311 & 2190 & 10434 & 1514 \\
\hline $\begin{array}{l}\text { Moderately preterm } \\
(n=20)\end{array}$ & 8581 & 889 & 5866 & 580 \\
\hline FC - fold change & & & &
\end{tabular}

In very preterm children, among the genes with a fold change $>2.0$, the expression of 536 probes ( 419 genes) decreased from birth to 36 weeks PMA and the expression of 1654 probes (1095 genes) increased between the two time points. No pathway was up-regulated at 36 PMA as compared to the cord blood and 1 pathway was down-regulated: hsa00590: Arachidonic acid metabolism.

In moderately preterm children, among the genes with a fold change $>2.0$, the expression of 146 probes (99 genes) decreased from birth to 36 weeks PMA and the expression of 743 probes ( 481 genes) increased between the two time points. One pathway was up-regulated at $36 \mathrm{PMA}$ as compared to cord blood (hsa04668: TNF signaling pathway) and two pathways were down-regulated (hsa04062: Chemokine signaling pathway; hsa04612: Antigen processing and presentation).

\section{Comparison of gene expression between subgroups of preterm infants}

Analysis of gene expression of the umbilical blood has shown no differences between the extremely preterm vs. moderately preterm groups and between the very preterm vs. moderately preterm groups. However, 88 probes (66 genes: 33 down-regulated and 33 up-regulated) were differentially expressed in the extremely preterm groups in comparison to the very preterm groups. No other pathways were differentially expressed.

Different situation was found at the $36^{\text {th }}$ PMA week. 4109 probes (3146 genes) were differentially expressed between extremely preterm and very preterm groups, 4856 probes ( 3405 genes) between extremely preterm and moderately preterm groups and 80 probes ( 54 genes) between very preterm and moderately preterm groups. However, the differences in expression of only a few genes were higher than twofold (Table 5).

Pathway analysis has shown no differences between the extremely preterm and very preterm groups, extremely preterm and moderately preterm groups and between the very preterm and moderately preterm groups.

\section{DISCUSSION}

In this study, we compared the results of whole genome expression profiles of cord blood and blood collected at the age of 36 weeks PMA from children born before 30 weeks of 
Table 5. Comparison of gene expression between subgroups of preterm infants

\begin{tabular}{|c|c|c|c|c|c|c|c|}
\hline & & \multicolumn{2}{|c|}{$\begin{array}{l}\text { Extremely preterm } \\
\text { vs. very preterm }\end{array}$} & \multicolumn{2}{|c|}{$\begin{array}{l}\text { Extremely preterm } \\
\text { vs. moderately preterm }\end{array}$} & \multicolumn{2}{|c|}{$\begin{array}{c}\text { Very preterm } \\
\text { vs. moderately preterm }\end{array}$} \\
\hline & & Probes & Genes & Probes & Genes & Probes & Genes \\
\hline $\mathrm{FC}>2.0$ & Up-regulated & 17 & 16 & 5 & 5 & 0 & 0 \\
\hline $\mathrm{FC}>2.0$ & Down-regulated & 13 & 12 & 431 & 292 & 0 & 0 \\
\hline
\end{tabular}

FC - fold change

gestation. The nature of the study was mainly exploratory, without any formal specific hypothesis to be verified. However, some predefined assumptions based on the selected group of patients can be made. Firstly, one can expect that preterm delivery would be associated with different gene expression profile in comparison to term delivery. Secondly, the gene expression profile may change with the maturity of a newborn measured by the gestational age. And finally, the expression of the genes in a referral time point represented by 36 PMA should be more or less similar for preterm babies.

There is a general agreement that term labor may be considered an inflammatory process that is associated with an increased expression of genes coding for pro-inflammatory cytokines, which starts the process of decidual activation. Localized inflammation leads to decidual disruption and might be one of the causes of preterm delivery. We considered this assumption as a sufficient rationale for our study. We were also aware that using cord blood for microarray analysis requires extrapolation of the results on the gene expression profile at the site of maternal fetal interphase.

\section{Comparison of gene expression profile between preterm subgroups vs. full term newborns}

The first observation is that there is a substantial difference between the gene expression profile at birth for infants born before 30 weeks of gestation and full-term children. Most of the genes in preterm newborns were under-expressed in comparison to the group of mature children. The difference in expression levels of more than $90 \%$ of these genes was only mildly to moderately significant (fold change $<2.0$ ). We performed detailed analysis of the genes with $\mathrm{FC}>2.0$ via the pathway enrichment analysis. This method can detect transcriptional programs that are distributed across an entire network of genes, yet are subtle at the level of individual genes. Using this method we found two pathways (Cytokine-cytokine receptor interaction and Chemokine signaling pathway), which were differentially regulated between both groups at the day of birth. Cytokine-cytokine receptor interaction pathway is responsible for intercellular signaling, controlling cell growth and differentiation, cell death, angiogenesis and host defense. Among the genes involved in this pathway are interleukins (IL1A, IL1B, IL23A, IL6, IL8, IL15), chemokines,
TNF and TNF receptors superfamily. During our study, we detected several genes from these pathways, which were down regulated in the preterm group versus the term babies. The study of expression of different CC and CXC chemokines in choriodecidual and maternal blood was performed in groups of term not in labor (TNF), term in labor (TL), idiopathic preterm labor (PTL) and preterm labor associated with infection (PTLI) [14]. Among differentially expressed chemokines, all of the groups revealed significant up-regulation in the TL vs. TNL. Comparison of PTL with TNL also showed an up-regulation of all the chemokines, except CCL16 (Fold change -14.4) and CCL19 (FC -1.8). It should be emphasized, however, that FC values for all up-regulated chemokines in these comparisons were significantly lower than those observed in TL vs. NTL (e.g.: 7.3 vs. 25.9; 27.2 vs. $35.1 ; 17.9$ vs. 44.5). Even, if authors did not perform a direct comparison of PTL vs. TL, based on the above results we can expect down regulation of chemokines in the PTL in comparison to the term labor (TL). This finding corroborates our results on the down-regulation of cytokine-cytokine receptor interactions and chemokine pathways in the PTL in comparison to TL. In the aforementioned study ten out of 12 CC and CX cytokines validated by PCR were significantly up-regulated in the TL vs. TNL group. The comparison of the preterm labor vs. term not in labor showed up-regulation in 5 chemokines (CCL4,5,8 and CXCL1,6), but only the CCL8 up-regulation was associated with the increased protein level. However, the level of significance for the up-regulation of PTL vs. TNL was 5 -fold lower in comparison with TL vs. TNL ( $p<0.05$ vs. $p<0.01$ ). Six chemokines did not show statistically significant differences. These also support the concept that a comparison with the TL should result in a down-regulation of the chemokine expression in the PTL. The analysis of chemokines in maternal plasma revealed that only CXCL8 chemokine was present in higher concentrations among mothers with TL vs. TNL and PTL vs. PTNL. CCL5 levels were higher in the PTL vs. PTNL but not vs. TL. CXCL10 was significantly higher in the TL vs. PTL. Generally, these results show a differential gene expression profile in the maternal decidua and maternal blood during preterm delivery, which does not contradict our results. There are, however, several important points that should be mentioned. Firstly, the sample size of the PTL was significantly 


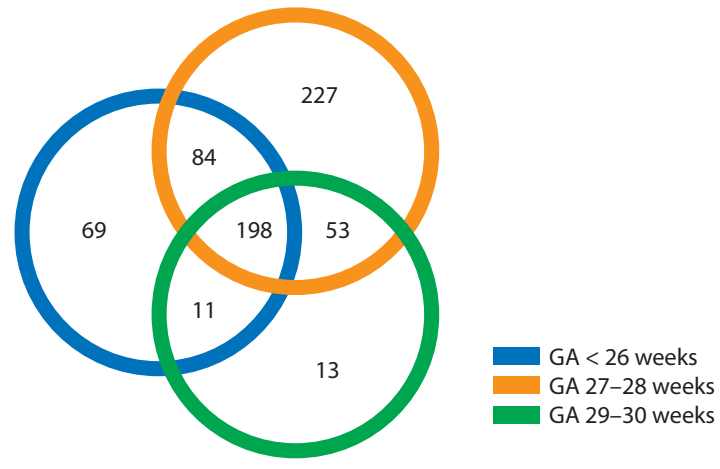

Figure 1. Comparison of the number of genes with decreased expression (fold change greater than 2.0 ) in the umbilical blood

lower than our group (20 vs. 62) and the authors did not provide any information about gestational age of preterm babies. Secondly, a high percentage of mothers in our preterm group received treatment with prenatal steroids (DxM) before delivery, which may inhibit the expression of chemokines in the cord blood. This was confirmed by the same authors who performed the analysis of chemokine expression profile in the choriodecidual explants treated with bethamethasone [14]. They found that CCL3, CCL4, CCL5, CXCL8 and CXCL10 were significantly down-regulated by dexamethasone treatment. Only CCL2 expression remained unaltered by the treatment. Finally, we can assume that both study groups show high preterm delivery etiologic heterogeneity, which is not easy to identify at the moment.

It was also interesting that among all the down regulated genes at the time of birth 198 genes were common for all three groups of preterm infants. The number of genes uniquely expressed by a specific gestational age group was as follows: GA $\leq 26$ wks (69), GA 27-29 wks (227) and GA 29-30 wks (13) (Figure 1). The common genes may represent determinants that are down-regulated in a manner that is not dependent on the gestational age. Inspecting the gene list reveals several interesting groups that can be identified:

- chemokine and ILR,

- G-protein signaling,

- long intergenic non-protein coding RNA,

- growth factors.

\section{Comparison of the gene expression profile at birth and at the age of 36 week PMA}

We found a large number of differentially expressed genes between the two time points. Analysis showed that the expression of about one third of the genome changed between birth and 6-12 weeks after birth. The difference in the expression fold change in the majority of genes ranged between 1.5 and 2.0, however, the expression of 285-1095 genes increased by twofold. The pathway analysis has shown 7 differentially regulated pathways between the two time-points.

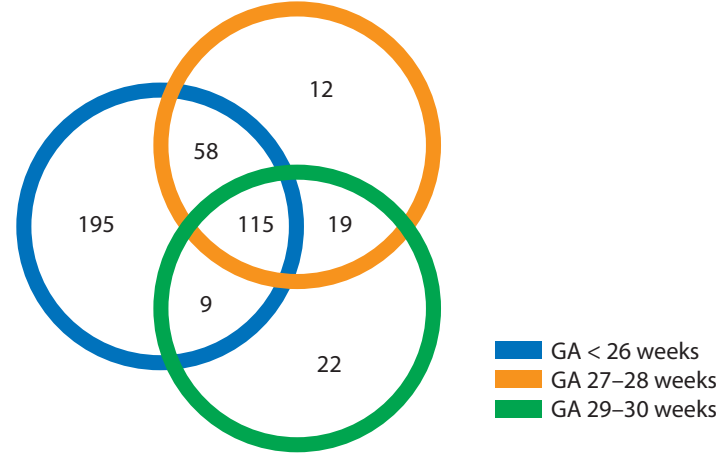

Figure 2. Comparison of the number of genes with increased expression (fold change greater than 2.0) at the 36 week postmenstrual age

Interestingly, there were more differentially expressed genes up-regulated in the group of preterm babies tested at the age of 36th PMA. The pathway analysis revealed only the TNF signaling pathway, which was repeated in extremely and moderately preterm groups. At the 36 PMA the group of up-regulated genes, that is common for three preterm categories, comprises 115 genes. However, among the genes specifically overexpressed in each GA category, the majority of genes were observed in the extremely preterm babies ( $n=195)$, whereas in the groups of very preterm and moderately preterm the numbers were significantly lower: $n=12$ and $n=22$, respectively (Figure 2). The pool of genes specific for the first group ( $G A \leq 26$ weeks) includes genes that are involved in:

- the mitotic cell activity represented by cyclin A2, cyclin dependent kinase, cell division cycle $34, \mathrm{G} 1$ to $\mathrm{S} 1$ transition,

- DNA repair mechanisms: growth arrest and DNA damage inducible alpha, RAD23 homolog A nucleotide excision repair protein,

- growth factors: growth differentiation factor, insulin like growth factor 2 mRNA binding protein, Kruppel like factor 1, BMP2 inducible kinase,

- mechanism of ubiquitination: ubiquitin $B$, ubiquitin conjugating enzyme E2, ubiquitin domain containing 1 . All these groups might reflect the processes representing acceleration of growth of the extremely immature infants, "trying" to catch up.

The numbers of genes, which were still down-regulated at the 36 PMA were 75, 83 and 55 for extremely, very and moderately preterm infants, respectively (Figure 3 ). A group of 32 down-regulated genes, shared by all the three groups, includes: the member of TNFR superfamily, chemokine receptor 5 and 6 and prostaglandin reductase. In comparison to the cord blood, there are no genes which continue to be down-regulated by the 36 PMA. Overall, the number of shared genes, which were down-regulated at birth, was reduced at the 36 PMA (from 198 to 32 ). It suggests that the 


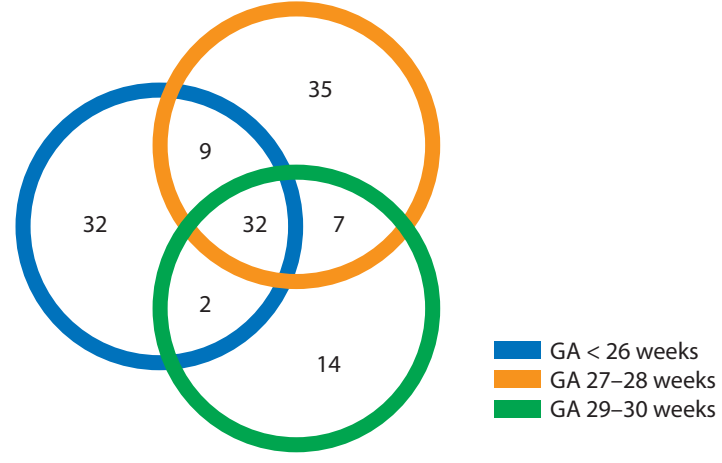

Figure 3. Comparison of the number of genes with decreased expression (fold change greater than 2.0) at the 36 week postmenstrual age

diminished number of down-regulated genes reflects the dynamic metabolic processes occurring between the birth and the 36 PMA and that at this age these children are approaching the maturity state of normal term babies. Based on the number of down-regulated genes, one can speculate that the extremely and very preterm babies still need more time to attain expression profile similar to the full term newborns. The fact that we were unable to find any specific pattern or pathway that would be significantly down-regulated at 36 PMA for any GA category, forefend building any reasonable hypothesis about specific mechanisms taking part in the maturation of preterm babies.

\section{Comparison of gene expression in the cord blood between subgroups of preterm infants}

Interestingly, we have not found any differences in gene expression between preterm infant subgroups in the cord blood. The explanation of this is challenging, especially considering that we have found a lot of differentially expressed genes in the blood collected at the $36^{\text {th }}$ week PMA. On the other hand, the difference was moderate and the expression of only a few genes was higher than twofold. These genes, however, did not show any specific pattern and no significant differentially expressed pathway was observed. A possible explanation is that the preterm labor is a very complex process associated with the interaction of many factors within a delicate network. Genes and gene products involved in this network might represent small effects, which are not sufficient to be "picked up" by rigorous statistical analysis. It may also imply that the gene expression profile during preterm delivery does not depend on the gestational age and represents a similar response to different extrinsic (e.g. infection) and intrinsic (maternal diseases, metabolic, stress etc.) trigger factors. The other probable explanation is that using only the cord blood may not reflect the true gene expression profile present in maternal tissue, such as decidua or placenta.
A Spanish group conducted a prospective cohort study comparing preterm (27-36 GA) or term (>37 wks) deliveries after a threatened preterm labour with term deliveries without suspected preterm labour [15]. They used placental samples for mRNA analysis and cord blood for selected protein inflammatory markers. Both groups of preterm delivery and term delivery after suspected preterm labour revealed an increased expression of TNF alpha, IL6 and PGF. The expression profiles of IFN gamma and VEGFR1 were decreased. Protein markers in the cord blood showed only an increased expression of TNF alpha and PGF. The other protein markers did not show any differences between the studied groups. This may suggest that expression profile in the cord blood does not fully reflect the profile observed in-situ [15].

An experimental study on fibroblast culture of term decidual cells obtained from uncomplicated term pregnancies after elective CS and before the onset of labor was performed by the group from The Ohio State University [16]. After fibroblast challenge with IL 1 beta for 6 hours up-regulation 448 transcripts and down-regulation of 116 transcripts was observed. Among highly up-regulated transcripts were pro-inflammatory cytokines (TNF, IL1A IL6), different chemokines and prostaglandin synthesizing enzymes. Pathway enrichment analysis provided cytokine-cytokine receptor interaction, apoptosis, Janus kinase signal transducer and STAT signaling [16]. This study supports the concept of up-regulation of genes involved in pro-inflammatory pathways at the time of delivery. It is, however, difficult to directly relate our results indicating on down-regulation of cytokine and chemokine related pathways to the above experimental studies because of the applied intervention with pro-inflammatory cytokine IL1 beta.

NICHD, NIH studies carried out by [17] focused on the transcriptome analysis of samples from chorio-amniotic membranes and blood taken from mothers at term with no labor (TNL) and term in labor (TIL) of uncomplicated pregnancy. The authors have found 197 transcripts which were significantly different between both groups. After gene ontology analysis TIL women revealed significant overexpression of multiple chemokines, neutrophil and monocyte recruitment pathways in chorioamniotic membranes. However, transcription profiling of the blood samples did not reveal any Gene Ontology enrichment category. This may indicate that human spontaneous labor without histologic signs of chorioamnionitis is characterized by a significant acute inflammatory response and that blood transcriptome may not fully correlate with in-situ gene expression profiling.

\section{CONCLUSIONS}

1. There is a substantial number of differentially expressed genes at the time of birth and at 36 PMA in compari- 
son with the term babies with more genes that are down-regulated than up-regulated.

2. Extremely and very preterm infants are characterized by significantly down-regulated cytokine and chemokine related pathways, which might reflect different preterm labor factors. Also, the effect of prenatal steroids cannot be excluded.

3. The decreasing number of down-regulated genes and the increasing number of up-regulated genes at 36 PMA vs. cord blood suggest that this is the time when preterm babies are approaching the state of maturity of term newborns. However, there is no specific pattern among these genes. Four down-regulated pathways, including arachidonic acid metabolism and one up-regulated (TNF signaling), may reflect the iatrogenic effects of intensive treatment of different morbidities, typically occurring in these groups of infants.

4. The lack of specific gene expression profile pathways within the groups of different GA may suggest activity of minor genes of small additive effects, rather than genes with major effects on the time of preterm delivery, which belong to the same pathway. This appears to be supported by the fact that all three groups shared nearly 200 down-regulated genes, whereas the groups of extremely and very preterm infants had 209 down-regulated genes in common, which represents factors that are not dependent on GA.

5. It should be kept in mind that the expression profile based on the cord blood may show weak correlation with the in-situ genes expressions.

\section{Acknowledgment}

The research leading to these results has been funded by the Polish-Norwegian Research Program operated by the National Centre for Research and Development under the Norwegian Financial Mechanism 2009-2014 in the frame of Project Contract No Pol-Nor/196065/54/2013.

\section{REFERENCES}

1. Stoll BJ, Hansen NI, Bell EF, et al. Eunice Kennedy Shriver National Institute of Child Health and Human Development Neonatal Research
Network. Trends in Care Practices, Morbidity, and Mortality of Extremely Preterm Neonates, 1993-2012. JAMA. 2015; 314(10): 1039-1051, doi: 10.1001/jama.2015.10244, indexed in Pubmed: 26348753.

2. Kusuda S, Fujimura M, Uchiyama A, et al. Neonatal Research Network, Japan. Trends in morbidity and mortality among very-low-birth-weight infants from 2003 to 2008 in Japan. Pediatr Res. 2012; 72(5): 531-538, doi: 10.1038/pr.2012.114, indexed in Pubmed: 22922774.

3. Beligere N, Perumalswamy V, Tandon M, et al. Retinopathy of prematurity and neurodevelopmental disabilities in premature infants. Semin Fetal Neonatal Med. 2015; 20(5): 346-353, doi: 10.1016/j.siny.2015.06.004, indexed in Pubmed: 26235349.

4. Jarjour IT. Neurodevelopmental outcome after extreme prematurity: a review of the literature. Pediatr Neurol. 2015; 52(2): 143-152, doi: 10.1016/j.pediatrneurol.2014.10.027, indexed in Pubmed: 25497122.

5. Strueby L, Thébaud B. Advances in bronchopulmonary dysplasia. Expert Rev Respir Med.2014;8(3):327-338, doi: 10.1586/17476348.2014.899907, indexed in Pubmed: 24666156.

6. Bhandari V, Bizzarro MJ, Shetty A, et al. Neonatal Genetics Study Group Familial and genetic susceptibility to major neonatal morbidities in preterm twins. Pediatrics. 2006; 117(6): 1901-1906, doi: 10.1542/peds.20051414, indexed in Pubmed: 16740829.

7. Saugstad O. Oxygen and oxidative stress in bronchopulmonary dysplasia. J Perinat Med. 2010; 38(6): 571-577, doi: 10.1515/jpm.2010.108.

8. Karna P, Muttineni J, Angell L, et al. Retinopathy of prematurity and risk factors: a prospective cohort study. BMC Pediatr. 2005; 5(1): 18, doi: 10.1186/1471-2431-5-18, indexed in Pubmed: 15985170.

9. Kim K, Zakharkin SO, Allison DB. Expectations, validity, and reality in gene expression profiling. J Clin Epidemiol. 2010; 63(9): 950-959, doi: 10.1016/j.jclinepi.2010.02.018, indexed in Pubmed: 20579843.

10. Slonim DK, Yanai I. Getting started in gene expression microarray analysis. PLoS Comput Biol. 2009; 5(10): e1000543, doi: 10.1371/journal. pcbi.1000543, indexed in Pubmed: 19876380.

11. Ritchie ME, Phipson B, Wu Di, et al. limma powers differential expression analyses for RNA-sequencing and microarray studies. Nucleic Acids Res. 2015; 43(7): e47, doi: 10.1093/nar/gkv007, indexed in Pubmed: 25605792.

12. Huang DaW, Sherman BT, Lempicki RA. Systematic and integrative analysis of large gene lists using DAVID bioinformatics resources. Nat Protoc. 2009; 4(1): 44-57, doi: 10.1038/nprot.2008.211, indexed in Pubmed: 19131956.

13. Huang DaW, Sherman BT, Lempicki RA. Bioinformatics enrichment tools: paths toward the comprehensive functional analysis of large gene lists. Nucleic Acids Res. 2009; 37(1): 1-13, doi: 10.1093/nar/gkn923, indexed in Pubmed: 19033363.

14. Hamilton SA, Tower $\mathrm{CL}$, Jones RL. Identification of chemokines associated with the recruitment of decidual leukocytes in human labour: potential novel targets for preterm labour. PLoS One. 2013; 8(2): e56946, doi: 10.1371/journal.pone.0056946, indexed in Pubmed: 23451115.

15. Oros $D$, Strunk $M$, Breton $P$, et al. Altered gene expression in human placenta after suspected preterm labour. Placenta. 2017; 55: 21-28, doi: 10.1016/j.placenta.2017.04.025, indexed in Pubmed: 28623969.

16. Ibrahim SA, Ackerman WE, Summerfield TL, et al. Inflammatory gene networks in term human decidual cells define a potential signature for cytokine-mediated parturition. Am J Obstet Gynecol. 2016;214(2):284.e1284.e47, doi: 10.1016/j.ajog.2015.08.075, indexed in Pubmed: 26348374.

17. Haddad R, Tromp G, Kuivaniemi H, et al. Human spontaneous labor without histologic chorioamnionitis is characterized by an acute inflammation gene expression signature. Am J Obstet Gynecol. 2006; 195(2): 394.e1394.24, doi: 10.1016/j.ajog.2005.08.057, indexed in Pubmed: 16890549. 